\title{
Modelling of Fires Following Bursts of Pressurized Fuel Tanks
}

G. M. MAKHVILADZE

Centre for Research in Fire and Explosion Studies,

University of Central Lancashire, Preston PR1 2HE, UK

\section{S. E. YAKUSH}

Institute for Problems in Mechanics, Russian Academy of Sciences, ave. Vernadskogo 101, Moscow 117526, Russia

\begin{abstract}
Formation and combustion of fuel clouds caused by failure of a pressurized vessel with hydrocarbon fuel is studied. Experimental data on expansion of instantaneously released clouds is analysed, a unified correlation for the expansion velocity as a function of time is obtained. A model for the turbulent cloud expansion following total loss of containment is developed, asymptotic behaviour of the cloud is compared with the experimental observations. The results of the theory are applied to formulation of initial conditions for numerical modelling of fireballs developing upon ignition of the fuel-air mixture after burst of a vessel with high-pressure gaseous fuel. Evolution and characteristics of the burning cloud are studied in a wide range of fuel masses and storage pressures. A unified correlation for the fireball lifetime is offered and compared with that for vertically directed fuel releases.
\end{abstract}

KEY WORDS : external fires, fire safety, hydrocarbon releases, fireballs, modelling

\section{INTRODUCTION}

Hydrocarbon fuels are being stored under high pressure on an increasing scale worldwide. Accidental loss of containment causes rapid mixing of fuel with the air and formation of a flammable cloud capable of exploding or burning as a fireball.

Pre-ignition development of fuel-air clouds following total loss of containment has, for the most part, been studied experimentally in the tests with bursting vessels filled with pressure-liquefied gases [1-4]. A number of experiments was also performed to measure the blast waves generated by the exploding vessels [5-7]. The experiments suggest that rapid expansion of compressed gases results in turbulization of the flow and intensive mixing of fuel with the ambient air. While the initial stage of cloud development is featured by propagation of shock waves, its further evolution is governed primarily by the turbulence created during the gas-dynamic stage.

Theoretical treatment of this phenomenon is still based on the early models $[1,8]$. The model [8] is based on the assumption of momentum conservation after directional release of fuel into the atmosphere and, thus, is not immediately applicable to almost spherically expanding clouds resulting from the total vessel breakdown. The model [1] only provides an approximating formula for the transient concentration distribution after vessel burst. None of these models can provide realistic initial conditions for the numerical modelling of fuel cloud combustion after total loss of containment.

CFD modelling of hydrocarbon fireballs was recently performed for short-duration vertical releases of single and two-phase fuels [9-12]. At the same time, modelling of fires following ignition of an instantaneously released fuel clouds has yet to be carried out. A 
crucial point in such modelling is posing adequate initial conditions which would reflect upon the characteristics of the turbulent cloud depending upon the fuel thermodynamic properties and pre-release storage conditions (i. e., whether or not the liquid is saturated or sub-cooled). Preliminary calculations have clearly shown that the numerical solutions are quite sensitive to the initial conditions, so that quantitatively and even qualitatively wrong results may be obtained if these conditions are posed arbitrarily.

This paper aims at developing a model for cloud expansion governed by its turbulent mixing with the air. The model is then applied to formulate the initial conditions for CFD modelling of fireball formation and development following total loss of containment with high-pressure gas (methane) and ignition of the resulting fuel cloud.

\section{ANALYSIS OF EXPERIMENTS}

Measurements of the visible cloud expansion velocities reported so far were performed for the bursting vessels filled initially with the pressure-liquefied gases. For a superheated liquid undergoing flash evaporation after a rapid drop in the pressure from its high value $P_{0}$ at the storage conditions to the ambient value of $P_{a}$, a characteristic velocity may be defined assuming that the expansion is isentropic, and that all the energy released in the flash evaporation is converted into the kinetic energy [8]:

$$
\frac{U_{*}^{2}}{2}=h_{l}\left(P_{0}\right)-\left(\left(1-x_{v}\right) h_{l}\left(P_{a}\right)+x_{v} h_{v}\left(P_{a}\right)\right)
$$

where $h_{l}$ and $h_{v}$ are the enthalpies of liquid and vapour on the saturation line, $x_{v}$ is the mass fraction of vapour determined from the isentropic relationship

$x_{v}=\frac{s_{l}\left(P_{0}\right)-s_{l}\left(P_{a}\right)}{s_{v}\left(P_{a}\right)-s_{l}\left(P_{a}\right)}$

(here $s_{l}$ and $s_{v}$ are the entropies of saturated liquid and vapour). The length and time scales for the expanding cloud are introduced as

$L_{*}=\left(\frac{M_{0} R_{g} T_{a}}{P_{a}}\right)^{1 / 3}, \quad t_{*}=\frac{L_{*}}{U_{*}}$.

In Fig. 1 the experimental data on the cloud expansion velocity $U_{b} / U_{*}$ (defined as the velocity of visible cloud edge) is plotted against the reduced time $t / t_{*}$, logarithmic scales are used on both axes. Points $1-4$ correspond to the experiments [1] with $M_{0}=0.125,1.95$, 15.6 and $452 \mathrm{~kg}$ of propylene. Points 5 represent the data from experiments with $4 \mathrm{~g}$ of Freon-113 carried out in [2]. Points 6 obtained in [3] using $0.74 \mathrm{~kg}$ of Freon-11. Points 7 are from the medium-scale tests [4] with $163 \mathrm{~kg}$ of propane.

It can be seen that, despite the big differences in the physical and thermodynamic properties of the substances, as well as in the scales of the experiments, all points follow the same trend which can be represented by a straight line in the logarithmic coordinates. The leastsquare fit of this dependency is

$$
\frac{U_{b}}{U_{*}}=0.25\left(\frac{t}{t_{*}}\right)^{-0.875} .
$$




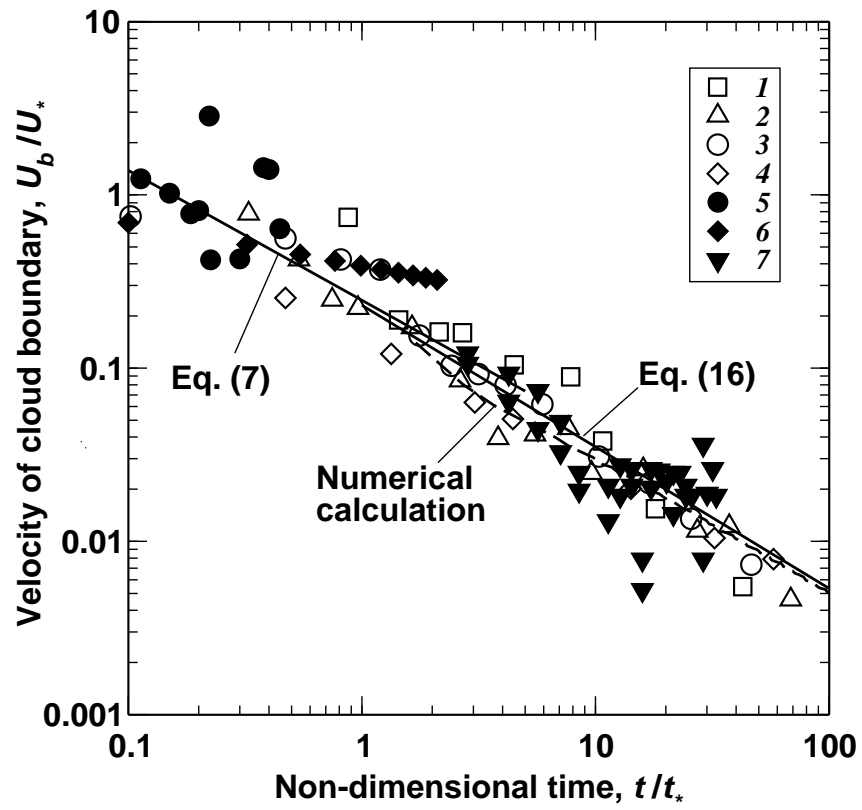

Fig. 1 - Summary of experimental data on cloud expansion velocity upon bursting of highpressure vessels..

It should be noted that the data is quite noisy, wide scatter of points exists not only between different experiments, but also within each separate test. This can be attributed to high intrinsic irregularity of the turbulent cloud and uncertainty in the initial conditions, boiling regime, bubble formation and breakup, etc. Also, cloud expansion is an essentially transient and short-duration process, which makes its accurate measurement difficult and could add to the data scatter.

Experimental studies of expanding single-phase clouds were mostly aimed at measuring the blast waves occurring in the atmosphere [5,6], while little has been reported about the concentration distributions and turbulent cloud growth. Explosive boiling of superheated liquid causes its breakup into a finely dispersed aerosol with the average droplet diameters of 10-100 $\mu \mathrm{m}$. When the expanding two-phase cloud mixes with the air, such droplets quickly evaporate, after which the cloud becomes purely gaseous. It is, therefore, reasonable to assume that at the later stages of evolution the non-dimensional characteristics of clouds from single-phase and two-phase releases should become similar, provided that appropriate scales are used.

Equation 2 for the length scale $L_{*}$ is based on the total fuel mass only and, thus, is applicable to both release types. The characteristic velocity of the single-phase cloud expansion may be determined from the isentropic expansion work $W_{i s}[13]$

$W_{i s}=\frac{P_{0} V_{0}}{\gamma-1}\left(1-\left(\frac{P_{a}}{P_{0}}\right)^{\frac{\gamma-1}{\gamma}}\right), \quad \frac{U_{*}^{2}}{2}=\frac{P_{0}}{\rho_{0}(\gamma-1)}\left(1-\left(\frac{P_{a}}{P_{0}}\right)^{\frac{\gamma-1}{\gamma}}\right)$, 
where $\gamma$ is the ratio of specific heats. The time scale is defined as in Eq. 2. These scales enable us to apply the cloud expansion relationship Eq. 3 to the analysis of both types of clouds.

Thus, the experimental data show that expansion of pressurized gas exhibits similarity in a wide range of substance properties and storage conditions. This similarity may be used to derive a mathematical model for the development of a turbulent cloud in the atmosphere after total loss of containment.

\section{MODEL FOR EXPANDING CLOUD}

Expansion of pressurized fuel clouds includes two stages, of which the first one is characterised by large radial velocities of gas driven by the pressure difference between the gas and the atmosphere. The duration of this gas-dynamic stage can be estimated as $L_{*} / c_{s}$ where $c_{s}$ is the speed of sound in the compressed gas. After the pressure waves travel far enough from the cloud, further evolution of the cloud is practically isobaric, and cloud expansion is governed by its mixing with the ambient air. At this second stage no significant velocities exist, so that no turbulence generation occurs. The viscous dissipation of turbulent energy and its decrease caused by mixing with non-turbulent ambient air results in the gradual decrease in the turbulent diffusivity and cloud propagation velocity.

Since the first stage of cloud expansion is driven by bulk gas motion, it is possible to apply at this stage, with some modifications, the model [8] based on the assumption of total momentum conservation. The modification has to be done because in the case of a radially expanding cloud the total momentum vector is zero due to spherical symmetry.

We consider the gas motion within some solid angle $d \Omega$ and assume that the total momentum of the gas confined to this solid angle remains constant, while the mass of moving gas increases with time due to frontal capture of the ambient air. By equating the total momentum created by the source within this solid angle, and the corresponding momentum of the cloud at some instant $t$, we obtain

$\frac{d \Omega}{4 \pi} M_{0} U_{0}=\frac{d \Omega}{3} R_{b}(t)^{3} \rho_{a} U_{b}(t)$,

where $R_{b}(t)$ is the cloud radius and $U_{b}(t)$ is the cloud expansion velocity. Since $U_{b}(t)=$ $d R_{b}(t) / d t$, an ordinary differential equation follows from Eq. 5:

$\frac{d\left(R_{b}^{4}\right)}{d t}=\frac{3 M_{0} U_{0}}{\pi \rho_{a}}$

which, after integration, gives the cloud radius and expansion velocity:

$R_{b}(t)=\left(\frac{3 M_{0} U_{0} t}{\pi \rho_{a}}\right)^{1 / 4}, \quad U_{b}(t)=\frac{1}{4}\left(\frac{3 M_{0} U_{0}}{\pi \rho_{a}}\right)^{1 / 4} t^{-3 / 4}$.

By relating the cloud radius and velocity to the scales Eq. 2, the following nondimensional relationships may be obtained:

$\tilde{R}_{b}(\tilde{t})=\left(\frac{3}{\pi}\right)^{1 / 4} \tilde{t}^{1 / 4}, \quad \tilde{U}_{b}(\tilde{t})=\frac{1}{4}\left(\frac{3}{\pi}\right)^{1 / 4} \tilde{t}^{-3 / 4}$. 
Here, we assumed that the initial gas velocity $U_{0}$ is equal to the characteristic velocity $U_{*}$. Thus, the integral model based on momentum conservation gives the law of expansion velocity decrease with time as $\tilde{t}^{-3 / 4}$. The cloud growth dependence following from this model is $\tilde{t}^{1 / 4}$. Note that a similar result has been earlier obtained by Roberts [14].

To describe the cloud expansion at the second stage we need to consider the turbulent kinetic energy $k$ and its dissipation rate $\varepsilon$. For simplicity we consider a spherical cloud of radius $R_{b}$ and assume top-hat profiles for all variables. The cloud expansion velocity $U_{b}=d R_{b} / d t$ is determined by the turbulent diffusion coefficient $D$. It is known that for an instantaneous point source of mass and constant diffusivity the characteristic radius of concentration distribution increases with time as $R_{b}=2(D t)^{1 / 2}$, the propagation velocity is then $d R_{b} / d t=(D / t)^{1 / 2}$. At the late stage of cloud expansion the gas density may be assumed constant and equal to the ambient density. Introducing the current cloud mass $M=$ $(4 / 3) \pi R^{3} \rho$ and using the volume-averaged equations for the kinetic energy and dissipation rate from the $k-\varepsilon$ model of turbulence [15], we obtain the following system of equations (all variables are made non-dimensional using the same scales as before, the scales for $k$ and $\varepsilon$ being $U_{*}^{2}$ and $U_{*}^{2} / t_{*}$ respectively):

$$
\begin{aligned}
& \frac{d \tilde{R}_{b}}{d \tilde{t}}=\left(\frac{\tilde{D}}{\tilde{t}}\right)^{1 / 2}, \quad \tilde{D}=\frac{C_{\mu}}{\operatorname{Sc}} \frac{\tilde{k}^{2}}{\tilde{\varepsilon}}, \\
& \frac{d \tilde{M} \tilde{k}}{d \tilde{t}}=-\tilde{M} \tilde{\varepsilon}, \\
& \frac{d \tilde{M} \tilde{\varepsilon}}{d \tilde{t}}=-C_{2} \tilde{M} \frac{\tilde{\varepsilon}^{2}}{\tilde{k}},
\end{aligned}
$$

where Sc is the Schmidt number, $C_{\mu}$ and $C_{2}$ are the constants of the $k-\varepsilon$ model of turbulence. To obtain the cloud behaviour at large times we seek for power-law solutions

$\tilde{R}_{b}=A \tilde{t}^{\alpha}, \quad \tilde{k}=B \tilde{t}^{\beta}, \quad \tilde{\varepsilon}=E \tilde{t}^{v}$.

Substituting these functions into the system of Eqs. (8)-(10) we obtain

$$
\begin{aligned}
& \alpha A \tilde{t}^{\alpha-1}=\left(\frac{C_{\mu} B^{2}}{\operatorname{Sc} E}\right)^{1 / 2} \tilde{t}^{\beta-(v+1) / 2}, \\
& (3 \alpha+\beta) B \tilde{t}^{3 \alpha+\beta-1}=-E \tilde{t}^{3 \alpha+v}, \\
& (3 \alpha+v) E \tilde{t}^{3 \alpha+v-1}=-C_{2} \frac{E^{2}}{B} \tilde{t}^{3 \alpha+2 v-\beta} .
\end{aligned}
$$

Balancing the left and right-hand sides in Eqs. (12)-(14), one obtains

$$
\beta=2(\alpha-1), \quad v=2 \alpha-3, \quad B=\frac{(2-5 \alpha) \alpha^{2} \mathrm{Sc}}{C_{\mu}} A^{2}, \quad E=(2-5 \alpha) B
$$


Substitution of these relationships into Eq. (14) leads to the following result:

$\alpha=\frac{2 C_{2}-3}{5\left(C_{2}-1\right)}$

The exponent $\alpha$ in the cloud growth law depends on the constant $C_{2}$ of the $k-\varepsilon$ model of turbulence for which the widely accepted value is $C_{2}=1.92$. This gives $\alpha=0.183$, while the exponents in the turbulent kinetic energy and dissipation rate decay laws are $\beta=-1.634$, $v=-2.634$. The cloud boundary velocity decreases with time as $t^{\alpha-1}$, which gives the power-law exponent $\alpha-1=-0.817$ quite close to that obtained from the experiments (Eq. 3).

It is interesting to find out the integral scale of turbulence using the results obtained:

$\tilde{\Lambda}=C_{\mu}^{1 / 2} \frac{\tilde{k}^{3 / 2}}{\tilde{\varepsilon}}=\frac{\alpha \mathrm{Sc}^{1 / 2}}{(2-5 \alpha)^{1 / 2}} A \tilde{t}^{\alpha}$.

One can see that the integral scale of turbulence grows proportionately to the cloud radius:

$$
\frac{\tilde{\Lambda}}{\tilde{R}_{b}}=\frac{\alpha \mathrm{Sc}^{1 / 2}}{(2-5 \alpha)^{1 / 2}} \approx 0.125 .
$$

This is consistent with experimental observations [1] where the macro-scale of turbulent vortices found from high-speed film records was shown to be $0.45 R_{C}$ where $R_{C}$ is the radius of cloud core in which half of the total mass of gas is concentrated, $R_{C} \approx 0.5 R_{b}$.

The constants $A, B$ and $E$ in Eq. 11 can not be found from Eqs. (8)-(10) because the initial conditions at the beginning of the second stage are uncertain, they are determined by flow turbulization during the gas dynamics stage. It is possible, however, to find the constant $A$ from the best fit of the function $\tilde{U}_{b}=\alpha A \tilde{t}^{\alpha-1}$ to the experimental data plotted in Fig. 1 . This gives $A=1.26$, after which the remaining constants may be found from Eq. 15 . The relationships for decay of expansion velocity and turbulent characteristics with time are

$\tilde{U}_{b}=0.23 \tilde{t}^{-0.817}, \quad \tilde{k}=0.46 \tilde{t}^{-1.634}, \quad \tilde{\varepsilon}=0.50 \tilde{t}^{-2.634}$.

The dependencies of cloud edge velocity on time resulting from the models implying momentum-dominated (Eq. 7) and turbulent diffusion-dominated (Eq. 16) mechanisms of cloud growth are shown in Fig. 1 by the straight lines. The intersection of these lines at $\tilde{t} \approx 1$ indicates transition between the corresponding cloud growth regimes. The model (8)(10) quantifies the turbulent characteristics of the cloud and may be used to pose adequate initial conditions for the numerical calculations.

\section{NUMERICAL MODELLING OF FIREBALL EVOLUTION}

\section{CFD Model}

The numerical model developed earlier for fireballs from vertical releases of hydrocarbons [9-12] is applied here to study transient characteristics of fireballs developing after total loss of containment.

The CFD model is based on a system of Favre-averaged mass, momentum, energy and species conservation equations closed by the $k-\varepsilon$ model of turbulence [15] and eddy break- 
up model of turbulent combustion. The gaseous phase consists of $\mathrm{O}_{2}, \mathrm{~N}_{2}$, fuel gas, $\mathrm{CO}_{2}$ and $\mathrm{H}_{2} \mathrm{O}$, soot formation/oxidation is calculated from the global kinetic scheme (see details in [11]). Temperature dependencies of the enthalpies and heat capacities of all components are allowed for. The model takes into account the radiative heat transfer, the absorptivity of hot combustion products is calculated from the Weighted-sum-of-grey-gases (WSGG) model. The radiative transfer equations are solved for individual grey gases, after which the total radiative source term is obtained by summation over all components. Since the numerical model is used at the stage where no significant pressure waves exist and the gas flow is essentially subsonic, the small Mach number approximation is used in which the gas is dynamically incompressible, but arbitrary density variation with temperature and chemical composition is allowed.

Calculations were performed for the initial fuel masses $M_{0}$ ranging from 1 to $1000 \mathrm{~kg}$, the initial pressure $P_{0}$ was also varied between 5 and 50 bar. Methane was used as the fuel, which makes the results obtained applicable to accidents at pressurised natural gas storage sites.

Each calculation started at an early time $t=t_{*}$, which corresponds to the non-dimensional time $\tilde{t}=1$. At this instant the cloud size and turbulence characteristics were specified according to the model (8)-(15). Top-hat profiles were used for the fuel mass fraction $Y$, temperature, kinetic energy $k$ and its dissipation rate $\varepsilon$ :

$\left\{\begin{array}{l}0 \leq r^{2}+z^{2} \leq R_{b}^{2}: Y=Y_{0}, \quad T=T_{0}, \quad k=k_{0}, \quad \varepsilon=\varepsilon_{0}, \\ r^{2}+z^{2}>R_{b}^{2}: \quad Y=0, \quad T=T_{a}, \quad k=k_{a}, \quad \varepsilon=\varepsilon_{a}\end{array}\right.$

The temperature $T_{0}$ and fuel mass fraction $Y_{0}$ inside the cloud were determined from the mass and energy balance assuming that the fuel undergoes adiabatic expansion and then mixes with some amount of air at the ambient temperature $T_{a}$. The gas velocity is set to zero and the pressure is assumed equal to the ambient $P_{a}$.

\section{Fireball Scales}

If a fuel-rich cloud is ignited shortly after loss of containment, it burns as a fireball rising due to its buoyancy and assuming nearly spherical shape. The dimensional analysis suggests that the maximum fireball size scales with the mass of fuel involved as $M^{1 / 3}$. It is shown in $[10,12]$ that the length, velocity and time scales relevant to burning clouds, are

$\hat{L}_{*}=\left(\frac{M_{0} Q}{\rho_{a} C_{p} T_{a}}\right)^{1 / 3}, \quad \hat{U}_{*}=\left(\hat{L}_{*} g\right)^{1 / 2}, \quad \hat{t}_{*}=\left(\hat{L}_{*} / g\right)^{1 / 2}$.

The measured maximum diameter of the burning cloud is very close to $\hat{L}_{*}$. The scaling for the fireball lifetime $t_{F B}$, however, depends on whether some characteristic velocity $U_{0}$ is present in the problem. If the fireball is purely buoyancy-controlled, its lifetime $t_{F B} \propto \hat{t}_{*}$, while for momentum-dominated clouds $t_{F B} \propto \hat{L}_{*} / U_{0}$. An approximating formula for intermediate regimes was offered in [16]. While for the directed releases of fuel the definition of characteristic velocity $U_{0}$ is quite straightforward (it is equal to the gas velocity in the source), for bursting vessels such a velocity can be defined as the characteristic velocity of gas expansion $U_{*}$ (Eqs. 1,4). The relative importance of buoyancy and momentum is 


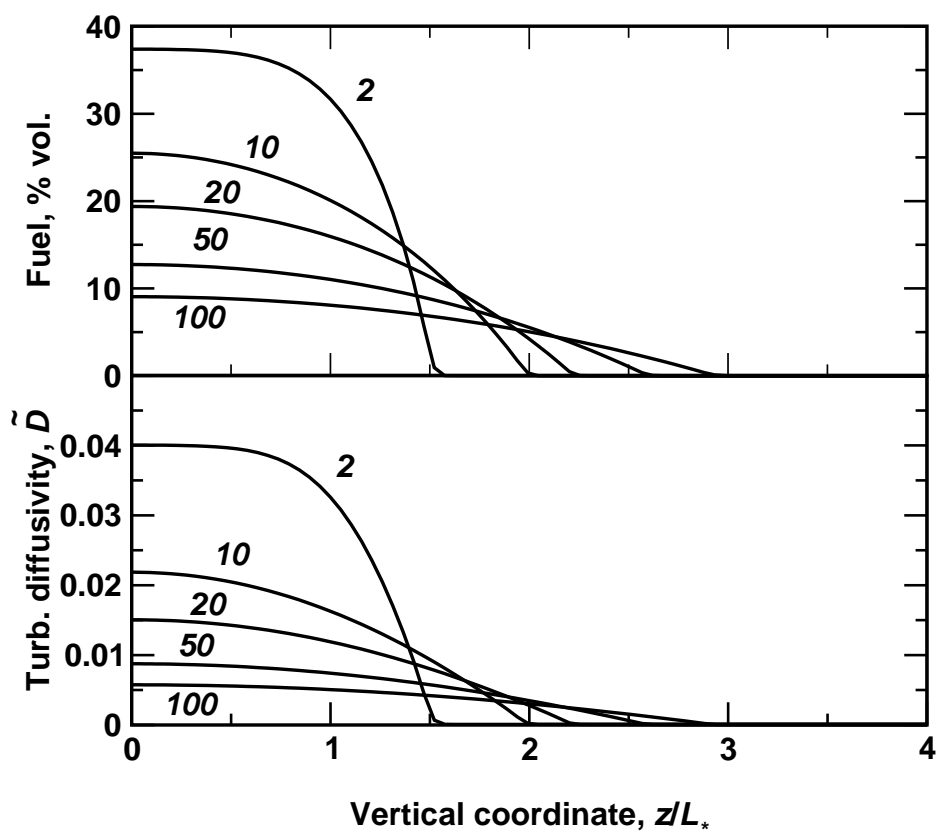

Fig. 2 - Expansion of turbulent cloud prior to ignition: profiles of fuel concentration and turbulent diffusivity are shown at indicated times $\tilde{t}$.

expressed in terms of the Froude number

$\operatorname{Fr}=\left(U_{*} / \hat{U}_{*}\right)^{2}$.

\section{Pre-ignition Cloud Development}

It is interesting to compare the dynamics of the cloud growth obtained numerically with the predictions of analytical model (Eq. 16). A calculation for $M_{0}=100 \mathrm{~kg}$ of methane stored initially at $T_{0}=T_{a}=293 \mathrm{~K}$ and $P_{0}=50 \mathrm{bar}$ was run (without ignition) up to $\tilde{t}=100$. The cloud expansion velocity was determined from the position of the contour on which the mass fraction of fuel is equal $10 \%$ of its maximum value. The calculated cloud expansion dynamics is presented in Fig. 1 by the dashed line. It can be seen that the non-dimensional expansion velocity tends with time to the line representing the cloud growth law (Eq. 16). Moreover, the calculated growth dynamics agrees with the experimental points in Fig. 1, which substantiates the proposed method of setting the initial conditions for calculation of turbulent clouds resulting from bursts of pressurised vessels.

In Fig. 2 the vertical profiles of volumetric fuel concentration and turbulent diffusivity in the expanding cloud are presented at several instants, demonstrating mixing of the cloud with the air and decay of turbulence with time. 


\section{Fuel Cloud Combustion}

Before igniting the fuel, the system was let to evolve for some period of time during which the cloud growth occurred exclusively due to turbulent mixing with the air. Typically, the ignition moment corresponded to $\tilde{t}_{i g}=5$. The ignition was performed by a hot kernel of the radius of $0.1 R_{*}$ placed at the centre of cloud. At the ignition instant the reaction within this kernel was promoted to completion, after which a combustion wave propagated through the cloud. Expansion of combustion products causes the cloud to grow radially, also, buoyancy forces generate the upward motion of the hot gas and its transformation into a fireball. The extent to which these two mechanisms affect the structure and shape of the burning cloud depends upon the Froude number Fr (Eq. 18). It is instructive to compare the results obtained for a fixed initial pressure $P_{0}$ (i. e., for constant $U_{*}$ ) and for two different fuel masses $M_{0}$. In this case variation of the Froude number is attributed to the change in the characteristic buoyancy-related velocity $\hat{U}_{*}$ (Eq. 17).

In Fig. $3 a-c$ the structure of burning cloud with fuel mass of $M_{0}=1 \mathrm{~kg}$ is shown at three consecutive instants $\hat{t}=0.23,0.50$ and $0.71(\tilde{t}=100,220$ and 310 , or, in the dimensional variables, $t=0.17,0.36$ and $0.52 \mathrm{~s}$ ). The storage pressure $P_{0}=50 \mathrm{bar}$, which corresponds to the characteristic expansion velocity of $U_{*}=688 \mathrm{~m} / \mathrm{s}$. The characteristic "buoyant" velocity is $\hat{U}_{*}=7.1 \mathrm{~m} / \mathrm{s}$ and the Froude number is $\mathrm{Fr}=9.3 \cdot 10^{3}$. At the first instant $50 \%$ of all fuel has been burnt, at the second one $95 \%$ of the fuel has reacted, while at the third one no fuel remains in the atmosphere. In each picture ten equally spaced temperature contours are shown, the minimum and maximum temperatures on the contours in Fig. $3 a, b$ are 400 and $1480 \mathrm{~K}$, while in Fig. $3 c$ they are 350 and $1250 \mathrm{~K}$ respectively. The radial and vertical coordinates are non-dimensionalized by the length scale $\hat{L}_{*}$. The velocity field is shown by arrows in each picture, the velocity scale is shown together with the corresponding maximum non-dimensional velocity value.

In Fig. $4 a-c$ similar contour maps are shown for a cloud with the fuel mass of $M_{0}=1000 \mathrm{~kg}$ at the times $\hat{t}=0.72,1.59$ and 2.23 (which corresponds to $\tilde{t}=100,220$ and 310, or, in the dimensional variables, $t=1.66,3.65$ and $5.15 \mathrm{~s}$ ). As before, the first two instants correspond to $50 \%$ and $90 \%$ fuel consumption. For this cloud the characteristic velocity $\hat{U}_{*}=22.5 \mathrm{~m} / \mathrm{s}$ and the Froude number is $\mathrm{Fr}=930$. The minimum and maximum temperatures on the contours in in Fig. $4 a, b$ are 400 and $1480 \mathrm{~K}$, while in Fig. $4 c$ they are 350 and $1250 \mathrm{~K}$ respectively. It can be seen that burning of the small-scale cloud is almost unaffected by buoyancy, the cloud remains hemispherical until all fuel has been consumed, and only then the products start to rise above the ground. The second cloud, however, by the time when fuel has been consumed, assumes the mushroom-like shape known as fireball.

It is interesting to compare the fireball lifetimes $t_{F B}$ in the cases of directed fuel releases (studied in [10]) and of spherically symmetric expansion of pressurized gas considered here. The fireball lifetime was defined as the time at which the total (integrated over the volume) reaction rate falls to $5 \%$ of its maximum value. The non-dimensional reciprocal burning times are shown against the square root of Froude number in Fig. 5. The open points are obtained for the storage pressure of $P_{0}=50$ bar and fuel masses $M_{0}=1,10$, 100 and $1000 \mathrm{~kg}$. The black points correspond to the same range of masses and the storage pressure of $P_{0}=5$ bar. It can be seen that all data collapse to the same linear correlation

$\hat{t}_{*} / t_{F B}=0.0178 \mathrm{Fr}^{1 / 2}$.

The dashed line shows the experimental correlation obtained for vertically directed short- 

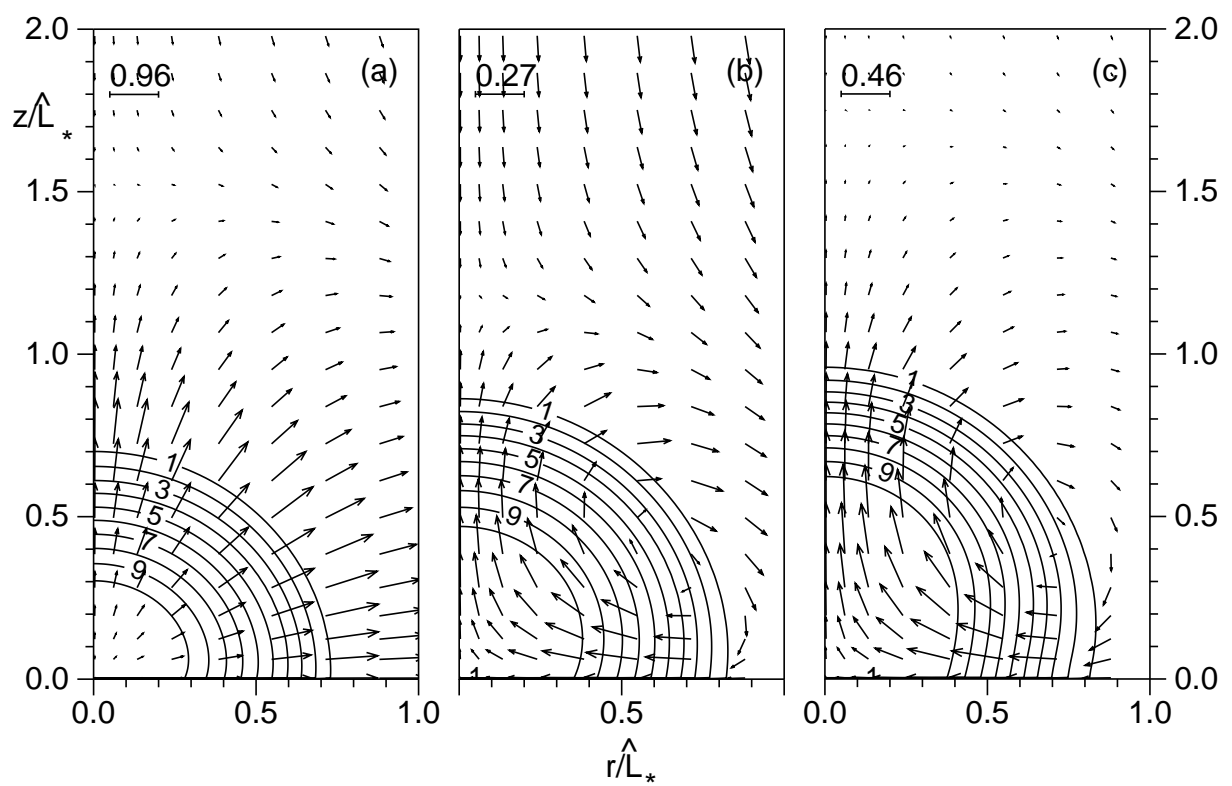

Fig. 3 - Fireball development for $M_{0}=1 \mathrm{~kg}:(a) \hat{t}=0.23$, (b) 0.50, (c) 0.71 .
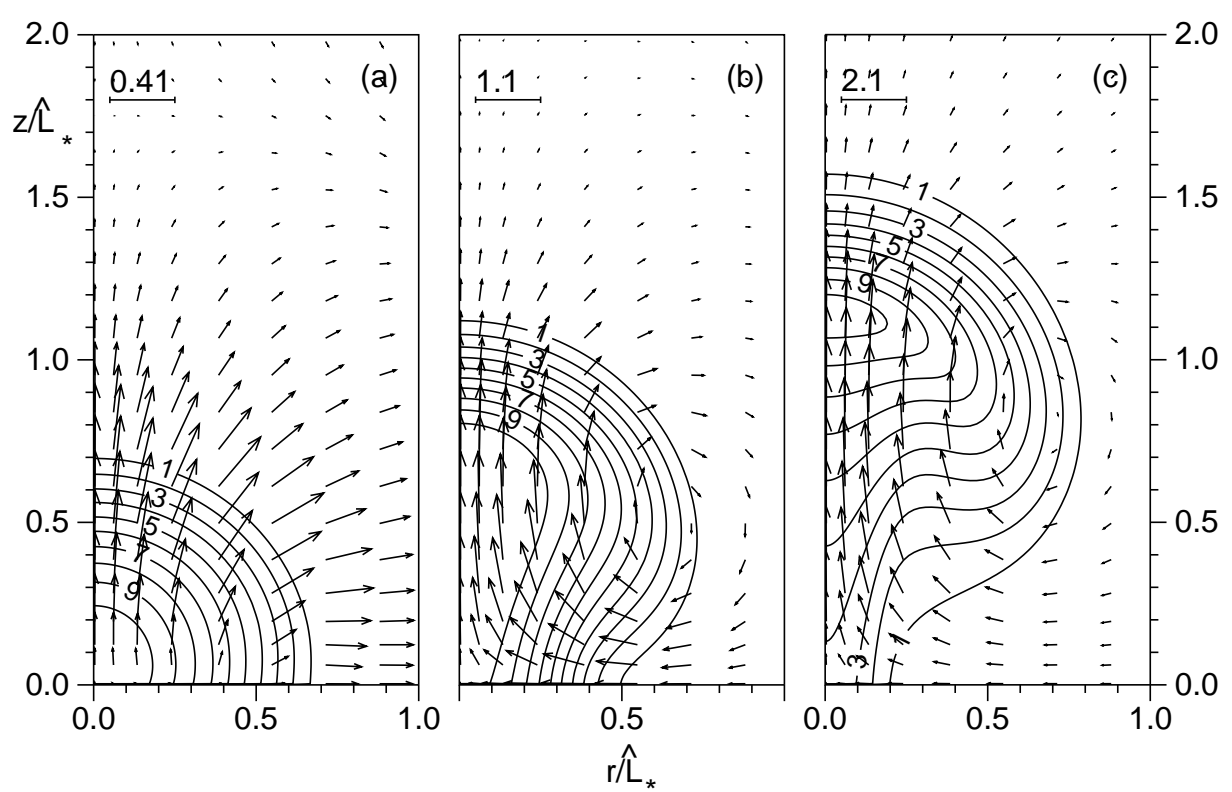

Fig. 4 - Fireball development for $M_{0}=1000 \mathrm{~kg}:(a) \hat{t}=0.72,(b) 1.59,(c) 2.24$. 


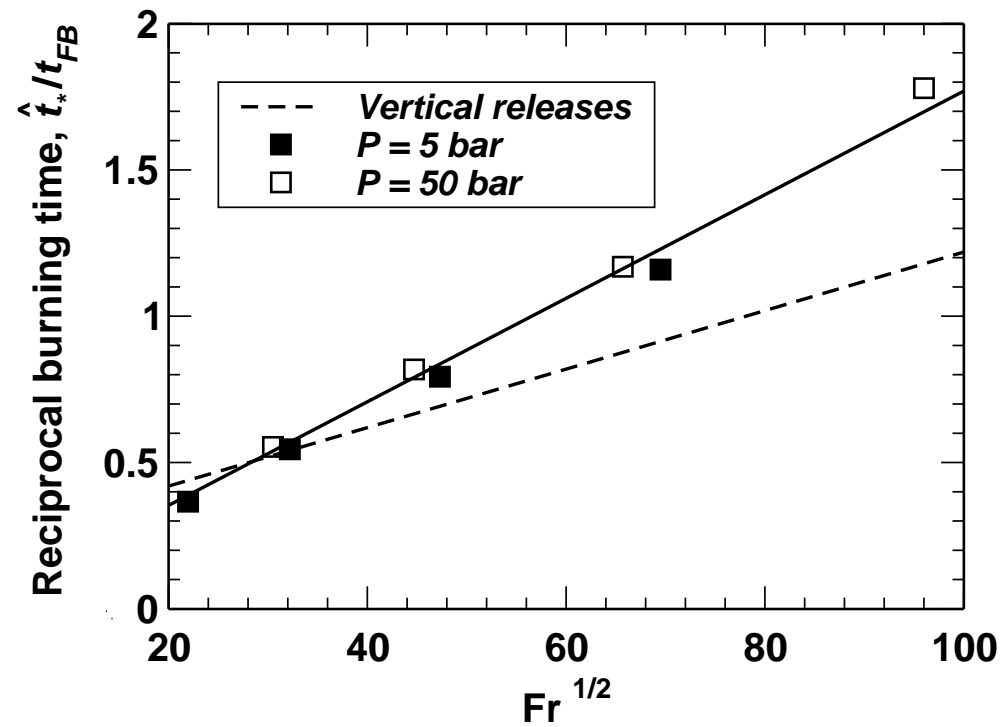

Fig. 5 - Fireball burning time dependence upon Froude number.

duration releases [16]:

$\hat{t}_{*} / t_{F B}=0.22+0.01 \mathrm{Fr}^{1 / 2}$.

Except for the largest clouds (i.e., the lowest Fr), the fuel clouds resulting from bursts of pressurised vessels burn faster than those released as a transient jet. This may be explained by higher turbulization and rapid mixing of the instantaneously released cloud with the air. The inverse proportionality between the fireball lifetime and the square root of the Froude number in Eq. 19 implies, according to the dimensional analysis, that the burning is controlled by the turbulence created by the gas expansion, rather than by gas buoyancy.

\section{CONCLUSIONS}

Experimental data on fuel cloud expansion following bursts of pressurized vessels are analysed, a power-law correlation for the cloud edge velocity as a function of time is obtained. Models for the momentum-dominated and turbulent diffusion stages of cloud expansion are presented, reasonable agreement of model predictions with the experimental data is demonstrated. Numerical studies of fuel cloud combustion following bursts of pressurized vessels revealed the structure of burning clouds for different Froude numbers. Large-scale clouds are more subject to influence of buoyancy, which causes their transformation into mushroom-like fireballs. Small-scale clouds burn out quickly enough, so that formation of vortex flow becomes evident only after total consumption of fuel. A correlation for the fireball burning time as a function of the Froude number is obtained and compared with the experimental relationship for vertically directed short-duration releases. The burning time for clouds generated by vessel bursts are shown to be shorter, which is attributed to higher intensity of turbulence created during gas expansion.

The authors would like to acknowledge the UK Engineering and Physical Sciences Research Council (Grant GR/M 18263) and INTAS (Grant 00-0706) for support of this study. 


\section{REFERENCES}

1. Giesbrecht, H., Hess, K., Leuckel, W., and Maurer, B., "Analysis of Explosion Hazards on Spontaneous Release of Inflammable Gases into the Atmosphere", Germ. Chem. Eng., 4:305-314 (1981).

2. Isaev, O. A., Nevolin, M. V., Skripov, V. P., and Utkin, S. A., "Expansion of Hot Liquid after Loss of Containment", J. Appl. Mech. Techn. Phys, 4:72-75 (1988).

3. Nolan, P. F., Pettitt, G. N., Hardy, N. R., and Bettis, R. J., "Release Conditions Following Loss of Containment”, J. Loss Prev. Process Ind., 3:97-103 (1990).

4. Maillette, J., and Birk, A. M., "Influence of Release Conditions on BLEVE Fireballs", ASME, Pressure Vessels and Piping Div., 333:147-152 (1996).

5. Esparza, E. D., and Baker, W. E., "Measurements of Blast Waves from Bursting Frangible Spheres Pressurized with Flash-evaporation Vapor or Liquid", Tech. Rep. CR2811, NASA, Washington, DC, 1977.

6. Baker, W. E., and Tang, M. J., Gas, Dust and Hybrid Explosions, Elsevier, Amsterdam, Oxford, New York, Tokyo, 1991.

7. Johnson, D. M., and Pritchard, M. J., "Large-scale experimental study of boiling liquid expanding vapour explosions (BLEVEs)", 14th Int. LNG/LPG Conference \& Exhibition, Gastech, 1990, pp. 1-30,

8. Hardee, H. C., and Lee, D. O., "Expansion of Clouds from Pressurized Liquids", Accid. Anal. Prev., 7:91-102 (1975).

9. Makhviladze, G. M., Roberts, J. P., and Yakush, S. E., "Modelling the Fireballs from Methane Releases", Fire Safety Science - Proceedings of the Fifth International Symposium, International Association for Fire Safety Science, 1997, pp. 61-81.

10. Makhviladze, G. M., Roberts, J. P., and Yakush, S. E., "Numerical Modelling of Fireballs from Vertical Releases of Fuel Gases", Combust. Sci. and Techn., 132:199-223 (1998).

11. Makhviladze, G. M., Roberts, J. P., and Yakush, S. E., "Combustion of twophase hydrocarbon fuel clouds released into the atmosphere", Combustion and Flame, 118:583-605 (1999).

12. Makhviladze, G. M., Roberts, J. P. and Yakush, S. E., "Modelling and Scaling of Fireballs from Single- and Two-phase Hydrocarbon Releases", Fire Safety Science Proceedings of the Fifth International Symposium, International Association for Fire Safety Science, Interscience Communications, London, UK, 2000, pp. 1125-1136.

13. Baker, W. E., Cox, P. A., Westine, P. S., Kulesz, J. J., and Strehlow, R. A., Explosion Hazards and Evaluation. Elsevier, Amsterdam, Oxford, New York, 1983.

14. Roberts, A. F., "Thermal Radiation from Releases of LPG from Pressurised Storage", Fire Safety Journal, 4:197-212 (1981/82).

15. Launder, B. E., and Spalding, D. B. Mathematical Models of Turbulence, Acad. Press, London, 1972.

16. Roper, F., Arno, J., and Jaggers, H. C., "The Effect of Release Velocity and Geometry on Burning Times for Non-premixed Fuel Gas Clouds", Combust. Sci. and Techn., 78:315-338 (1991). 